\title{
Saúde Mental na Atenção Básica: Análise das Práticas de Apoio Matricial
}

\section{na Perspectiva de Profissionais}

\author{
Larissa Moraes Moro* \\ Pontifícia Universidade Católica do Rio Grande do Sul - PUCRS, Porto Alegre, RS, Brasil \\ ORCID: https://orcid.org/0000-0001-7962-4577 \\ Guilherme Severo Ferreira** \\ Pontifícia Universidade Católica do Rio Grande do Sul - PUCRS, Porto Alegre, RS, Brasil \\ ORCID: https://orcid.org/0000-0003-3445-429X \\ Kátia Bones Rocha*** \\ Pontifícia Universidade Católica do Rio Grande do Sul - PUCRS, Porto Alegre, RS, Brasil \\ ORCID: https://orcid.org/0000-0001-7603-1709
}

\section{RESUMO}

Este estudo objetiva analisar os processos de trabalho em saúde mental na perspectiva de profissionais da Atenção Básica (AB) de Porto Alegre/RS, estabelecendo aproximações e distanciamentos nas práticas desenvolvidas, conforme a modalidade de apoio matricial: equipes de referência de saúde mental e equipes de NASF-AB. Estudo qualitativo transversal, realizado em oito serviços, na qual participaram 20 profissionais. Entrevistas semiestruturadas foram conduzidas e analisadas através da Análise Temática. Analisou-se que as equipes de NASF se mostraram mais presentes nas unidades, o que contribui para a construção de ações conjuntas com as Equipes de Saúde da Família. O apoio matricial mostrou ter efeitos na diminuição dos encaminhamentos, na maior participação das equipes nas ações de saúde mental e no aumento da resolutividade da atenção. Destaca-se a importância do estabelecimento de medidas e ações que busquem consolidar todas os tipos de equipe de apoio matricial enquanto estratégia de qualificação da atenção à saúde mental na $\mathrm{AB}$.

Palavras-chave: saúde mental, apoio matricial, atenção básica.

\section{Mental health in Primary Health Care: analysis of matrix support practices}

\section{from the perspective of professionals}

\begin{abstract}
This study aims to analyze the work processes in mental health from the perspective of professionals of Primary Health Care (PHC) of Porto Alegre/RS/Brazil, establishing similarities and differences in the practices developed according to the mode of matrix support: reference teams of mental health and Family Health Support Team (NASF). This qualitative and transversal study was performed in eight units, with the participation of 20
\end{abstract}

ISSN $1808-4281$ 
professionals. Semi-structured interviews were conducted which were analyzed through Thematic Analysis technique. It was analyzed that the NASF teams were more present in the units, which contributes to the construction of joint actions with the Family Health Teams. Matrix support has shown to have an effect on the reduction of referrals, on the greater participation of teams in mental health actions and on the increase of the resolution of care. The importance of establishing measures and actions that seek to consolidate all types of matrix support team as a strategy for qualifying mental health care in PHC is highlighted.

Keywords: mental health, primary health care, matrix support.

\title{
Salud mental en Atención Primaria: análisis de las prácticas de matriz de
}

\author{
soporte desde la perspectiva de los profesionales
}

\section{RESUMEN}

Este estudio tiene como objetivo analizar los procesos de trabajo en salud mental desde la perspectiva de los profesionales de la Atención Primaria de Salud (APS) de Porto Alegre, estableciendo similitudes y diferencias en las prácticas desarrolladas de acuerdo con la modalidad de matriz de soporte: equipos de referencia de salud mental y equipos del Núcleo de Apoyo a la salud de la Familia (NASF). Estudio cualitativo transversal, realizado en ocho unidades de salud, en la que participaron 20 profesionales. Se realizaron entrevistas semiestructuradas que fueron analizadas a través del análisis temática. Se analizó que los equipos NASF estaban más presentes en las unidades, lo que contribuye a la construcción de acciones conjuntas con los equipos de salud familiar. El matriz de soporte ha demostrado tener un efecto en la reducción de derivaciones, en la mayor participación de los equipos en las acciones de salud mental y en el aumento de la resolución de la atención. Se destaca la importancia de establecer medidas y acciones que busquen consolidar todo tipo de equipo de matriz de soporte como estrategia para calificar la atención de salud mental en APS.

Palabras clave: salud mental, matriz de soporte, atención primaria de salud.

O modelo de atenção em Saúde Mental brasileiro passou por mudanças fundamentadas na substituição de um modelo asilar por um enfoque baseado na atenção psicossocial. Assim, conforme a legislação da Lei 10.216, o cuidado em saúde mental deve ser prestado prioritariamente na comunidade em que a pessoa vive, fazendo uso de todos os recursos disponíveis (Lei n. 10.216, 2001).

Com o intuito de desenvolver a articulação dos serviços de atenção à saúde mental, a partir da Portaria $n^{\circ} 3.088$ instituiu-se a Rede de Atenção Psicossocial (RAPS). A RAPS reforça e destaca a função da Atenção Básica $(\mathrm{AB})$ na rede, enquanto ponto estratégico de cuidado em saúde mental no território (Portaria n. 3.088, 2011; Brasil, 2013). A RAPS visa 
articular e fortalecer as redes de atenção à saúde humanizadas e centradas nas necessidades das pessoas, pautadas no respeito aos direitos humanos, na garantia de autonomia e liberdade (Portaria n. 3.088, 2011).

Assim, a $\mathrm{AB}$ cumpre um papel estratégico nas redes de atenção, servindo como base para o seu ordenamento e para a busca da integralidade do cuidado. Nesse sentido, a Estratégia de Saúde da Família (ESF) apresenta potencial para desenvolver ações de cuidado em saúde mental, justificado pelo trabalho pautado em equipe multidisciplinar, pela inserção do agente comunitário de saúde, na adscrição dos usuários e na participação social. Uma dessas ações consiste na oferta de atendimento e tratamento na $\mathrm{AB}$ ou, quando necessário, no encaminhamento para serviços especializados (Brasil, 2013).

O Ministério da Saúde tem apontado como prioridade a qualificação da AB por meio da ESF, bem como a formulação e implementação de políticas neste sentido. Dentre elas, destaca-se a criação do Núcleo de Apoio à Saúde da Família (NASF), pela portaria n. 154, de 2008, que tem como objetivo ampliar a abrangência das ações da AB. A função do NASF é apoiar o trabalho desenvolvido pela ESF por meio de atendimento compartilhado, além de atuar de forma integrada por meio de equipe multidisciplinar, proporcionando o acompanhamento longitudinal do usuário no SUS (Portaria n. 154, 2008). Em 2017, com a Portaria n. 2.436, houveram alterações na Política Nacional da Atenção Básica (PNAB), dentre elas as diretrizes do NASF, alterado o termo para Núcleo Ampliado de Saúde da Família e Atenção Básica (NASF-AB). A política vigente apresenta como mudança a inclusão da abrangência das equipes NASF para todos os serviços da AB além da ESF (Portaria n. 2.436, 2017).

Uma estratégia que vem sendo incorporada pelos serviços a nível nacional para qualificar a atenção à saúde mental na AB é o Apoio Matricial (AM). Na RAPS, o AM atua como um arranjo organizacional que presta suporte técnico especializado em áreas específicas às equipes de saúde das unidades, a partir do pressuposto de responsabilização compartilhada. Além disso, tem como intuito a superação da lógica do encaminhamento, visando aumentar a capacidade resolutiva de problemas de saúde mental na AB (Brasil, 2013).

$\mathrm{O}$ apoio matricial em saúde mental, no contexto da $\mathrm{AB}$, tem revelado aspectos facilitadores do trabalho em rede, contribuindo com melhorias na articulação da RAPS e na integralidade do cuidado a partir da corresponsabilização entre os serviços (Onocko-Campos et al., 2012; Costa, Lima, Silva, \& Fioroni, 2015; Castro, Oliveira, \& Campos, 2016; Lima \& Dimenstein, 2016; Tatmatsu \& Araújo, 2016). Outros achados se referem a potencialidade que as atividades de AM têm de promover uma atuação interdisciplinar, maior sensibilização 
dos profissionais da $\mathrm{AB}$ com relação à demanda de saúde mental, a contrução de estratégias de intervenção no território (Lima \& Dimenstein, 2016; Tatmatsu \& Araújo, 2016; Amaral, Torrenté, Torronté, \& Moreira, 2018).

Contudo, também são evidenciados na literatura diversos impasses na efetivação do AM enquanto estratégia. Dentre eles, destacam-se: a lógica de trabalho dos serviços ainda pautada no modelo hegemônico biomédico (Morais \& Tanaka, 2012; Alvarez, Vieira, \& Almeida, 2019; Eslabão et. al, 2019); a dificuldade dos profissionais, tanto do nível primário como da atenção especializada de adotar essa metodologia de trabalho (Alvarez, Vieira \& Almeida, 2019; Gerbaldo, Arruda, Horta, \& Garnelo, 2018; Klein \& Oliveira, 2017; Treichel, Onocko-Campos, \& Campos, 2018); além da desvalorização do AM pelos gestores e trabalhadores de saúde (Machado \& Camatta, 2013; Tatmatsu \& Araújo, 2016).

Nesse sentido, existe um consenso na literatura quanto à necessidade de avanços na Reforma Psiquiátrica brasileira, destacando a premência de haver interligação entre redes assistenciais baseadas no AM, com o intuito de favorecer um cuidado integral ao usuário (Bonfim, Bastos, Góis, \& Tófoli. 2013; Gerbaldo, Arruda, Horta, \& Garnelo, 2018; Lima \& Dimenstein, 2016). As pesquisas reiteram a necessidade de fortalecer a $A B$ como via de acesso e de articulação da rede, garantindo o cuidado continuado dos sujeitos e uma efetiva resolutividade da atenção. Ademais, os autores destacam a emergência de avanços no cuidado na $\mathrm{AB}$, principalmente em relação à capacitação dos profissionais e no aumento de recursos de AM (Gerbaldo, Arruda, Horta, \& Garnelo, 2018; Lima \& Dimenstein, 2016).

Diante desse cenário, percebe-se a necessidade de conhecer as ações ofertadas na $\mathrm{AB}$, bem como as práticas e estratégias que podem ser produzidas pelos serviços para que a demanda em saúde mental encontre resolutividade na RAPS. Considerando que o NASF é ainda uma política recente e encontra-se em processo de implantação, construção e efetivação das suas práticas, evidencia-se a importância de analisar como vem sendo percebidas as ações de apoio matricial em saúde mental nas unidades. Diante do exposto, este estudo tem como objetivo analisar os processos de trabalho em saúde mental a partir da perspectiva dos profissionais da $\mathrm{AB}$ de Porto Alegre, tecendo aproximações e distanciamentos nas práticas desenvolvidas conforme a modalidade de AM recebida pelas unidades: de equipe de referência de saúde mental e de equipe NASF. 


\section{Método}

Trata-se de um estudo qualitativo, transversal e de caráter exploratório. O cenário pesquisado foi Porto Alegre/RS, a qual conta com oito gerências distritais de saúde. Dessas, cinco gerências de saúde continham equipes de NASF atuando nos serviços da AB. Assim, foram sorteadas duas unidades de cada gerência, uma unidade com apoio matricial do NASF, e uma com apoio matricial de equipe de referência de saúde mental, totalizando dez unidades. Não foi possível realizar a coleta em duas unidades; um serviço não aceitou participar da pesquisa e no outro houve indisponibilidade de horário para receber os pesquisadores. A coleta de dados foi realizada em oito unidades de saúde, sendo uma Unidade Básica de Saúde (UBS) tradicional e sete unidades com Equipes de Saúde da Família (ESF).

Em relação à rede da cidade pesquisada, conforme o Plano Municipal de Saúde (20182021), existem 143 unidades de saúde da AB, sendo 88 serviços ESF e 55 serviços UBS. A rede compreende diferentes modalidades de equipes de apoio matricial. Exitem 22 equipes de saúde mental (adulto e infantil), que atuam por meio de equipes interdisciplinares e são referência para os demais serviços da rede das regiões onde estão situadas. $\mathrm{O}$ acesso se dá a partir da $\mathrm{AB}$ e da atenção especializada pelos CAPS. A outra categoria que realiza AM em saúde mental são os NASF. No momento de realização da pesquisa contabilizavam-se sete equipes NASF cadastradas (CNES, 2017).

Participaram da pesquisa 20 profissionais, tendo como critério de inclusão ser trabalhador da equipe da $\mathrm{AB}$ e ter interesse em participar do estudo. Os sujeitos participantes foram: oito enfermeiros, seis agentes comunitários de saúde e seis médicos. No intuito de facilitar o entendimento a respeito das diferentes equipes de apoio matricial, os profissionais foram organizados em dois grupos: Grupo 1, o qual compreende dez profissionais de unidades com apoio matricial de equipe de saúde mental; e Grupo 2, que contempla dez profissionais de unidades que contavam com apoio matricial de equipe NASF.

Como instrumento de coleta de dados, foram utilizadas entrevistas semiestruturadas, com roteiro que abordou os processos de trabalho relacionados à atenção à saúde mental e percepção do apoio matricial na $\mathrm{AB}$. As entrevistas foram realizadas por dois pesquisadores, no local de trabalho dos profissionais, com dia e horário pré-agendados por telefone. As entrevistas tiveram duração aproximada de 40 minutos, as quais foram gravadas e transcritas na íntegra. A identidade dos participantes foi preservada e as falas foram identificadas como P1, P2 e assim por diante, seguidos da categoria profissional e o grupo categórico pertencente. 
Para a análise do material, adotou-se a técnica de Análise Temática, segundo Braun e Clarke (2006). Os passos seguidos no procedimento de análise foram: (a) familiarização dos dados (transcrição e leitura das entrevistas); (b) codificação inicial dos temas e padrões recorrentes; (c) escolha e definição dos temas (análise e relação entre códigos); (d) releitura e revisão dos temas; (e) Definição e nomeação dos temas, (f) redação analítica, interligando extratos de falas mais significativos dos temas. Os procedimentos de codificação e tematização dos dados foram realizados por dois pesquisadores com o auxílio do programa Atlas TI.

As entrevistas foram realizadas após a leitura e assinatura do Termo de Consentimento Livre e Esclarecido, que foi disponibilizado em duas cópias. Essa pesquisa foi aprovada pelos Comitês de Ética em Pesquisa (CEP) e da Secretaria Municipal de Saúde de Porto Alegre (sob número de parecer 1.540.814).

\section{Resultados e Discussão}

Os resultados apresentados objetivam caracterizar os temas mais relevantes e frequentes referentes às práticas de saúde mental na $\mathrm{AB}$ e aos atravessamentos do apoio matricial em saúde mental nesse processo. A partir da análise de dados proposta, foram geradas 13 unidades de códigos, os quais foram agrupados por temas congruentes entre si. Por sua vez, os temas mais evidentes e significativos foram organizados em categorias finais. Diante disso, resultaram desse processo quatro categorias de análise: Dinâmicas de encaminhamento; Ofertas de atendimento em saúde mental; Ações de Apoio Matricial em saúde mental; e Avaliação dos profissionais sobre o Apoio Matricial.

\section{Dinâmicas de Encaminhamento da Atenção Básica}

Ao questionar sobre como eram tomadas as decisões de encaminhar usuários para a atenção especializada, encontrou-se uma variedade de percepções sobre os critérios utilizados por cada profissional e equipe. Os critérios mais mencionados foram: gravidade de cada caso, urgência, questões sintomáticas agudas e situações que envolvem o risco à vida do usuário (tentativas de suicídio) ou de outras pessoas. Quando tais questões são avaliadas, há um consenso entre os profissionais de que se faça o encaminhamento diretamente para os serviços de emergência da cidade, para depois disso, continuar o tratamento na unidade básica. Esses aspectos foram relatados por profissionais de ambos grupos: 
Quando envolve risco de vida, não tenho como esperar. Quando é uma situação aguda, que envolva risco para si ou risco para outrem, é emergência psiquiátrica. Quando a gente vê uma necessidade aguda, grave, a gente remete, para compensar. Depois, então, começar a fazer a abordagem por aqui (P13 - enfermeira/grupo 2).

Além disso, também foi abordado como são decididos os casos passíveis de encaminhamento. Nesse ponto, encontraram-se distinções entre as narrativas dos profissionais dos dois grupos. Os trabalhadores de unidades com apoio NASF (grupo 2), relataram que as decisões e os parâmetros são avaliados de forma coletiva, com a opinião de mais de um profissional, enquanto os entrevistados de serviços com apoio de equipe de saúde mental (grupo 1) mencionam que a decisão de encaminhamento é perpassada preponderantemente pela posição do médico da unidade.

Aquele paciente que chega aqui com depressão profunda, não toma banho, não quer comer, triste, fala pouco, não te olha na cara, se despreocupa com aspectos pessoais. Eu atendo, eu avalio, mas eu peço em equipe os critérios das outras pessoas (P.2 médico da família, grupo 2).

Uma das possíveis razões para que isso ocorra encontra-se no fato de que as equipes NASF realizavam o trabalho in loco nos serviços, ao passo que as equipes de saúde mental ficavam predominantemente nos respectivos serviços de referências. Dessa forma, quando há casos para serem discutidos, frequentemente são os médicos das unidades que vão às reuniões de matriciamento com as equipes de saúde mental. Em relação ao matriciamento das equipes de saúde mental, destaca-se a seguinte fala:

O matriciamento também prevê isso, que os profissionais circulem pelas equipes diretamente ou através dessa parte de educação continuada. Devido até essa quantidade reduzida de profissionais em saúde mental, eles ficam mais no local deles, atendendo o que chega. Eles não podem sair e trocar (P.11 - enfermeira, grupo 1).

Nos locais do grupo 2 foram relatadas duas situações: alguns profissionais mencionaram que os médicos são responsáveis pela exposição dos casos considerados mais 
graves nas reuniões de matriciamento, e outros comentaram sobre a participação de toda a equipe nos momentos de reunião com NASF. Essas visões podem ser observadas nos trechos:

Geralmente qualquer profissional da equipe pode ficar responsável de matriciar um caso. Tanto um agente de saúde, enfermagem ou um médico. Porém, normalmente esses casos mais graves são do médico, né. (P.4 - enfermeira, grupo 2).

Para ter um olhar diferenciado sobre cada caso, a gente tem as nossas reuniões de matriciamento, que são multidisciplinares. É toda equipe juntamente com o NASF. A gente disseca o caso, cada um traz a sua contribuição do que conhece daquela família, daquele indivíduo, para tentar entender melhor o processo que ele está passando e como a gente vai direcionar essa família ou sujeito para aquilo que ele necessita (P.13 - enfermeira, grupo 2).

O segundo trecho de fala denota que o trabalho do NASF nos territórios dos serviços favorece a integração entre as equipes, fazendo com que todos tenham a possibilidade de participar das ações de matriciamento na unidade, o que pode ser compreendido como um facilitador no modo corresponsável e compartilhado de encaminhamento. Porém, nota-se que em algumas situações ainda há uma disparidade em relação à participação do médico e demais profissionas nas reuniões com a equipe NASF.

Ressalta-se que o Ministério da Saúde (Brasil, 2013) propõe que a $A B$ realize encaminhamento para a atenção especializada quando as demandas não podem ser atendidas nas unidades básicas, por exemplo, quando há a presença de sintomas mais graves, como tentativa de suicídio, alucinações e uso abusivo agudo de substãncias. Pressupõe-se que o processo de encaminhamento seja implicado, o qual o profissional se corresponsabiliza e participa ativamente de todo o processo de chegada do usuário a outro serviço (Brasil, 2013). A responsabilização que envolve o encaminhamento dos casos e orienta o fluxo de serviços na rede deve pressupor a continuidade do cuidado em todo percurso terapêutico. A corresponsabilização é a implicação dos diversos sujeitos envolvidos numa prática que considere aspectos singulares dos sujeitos, valorização e preocupação com o cuidado (Bezerra, Jorge, Gondim, Lima, \& Vasconcelos, 2014).

Na verdade, a decisão de encaminhar é junto ao NASF. A nossa primeira intenção é sempre atender aqui. A gente não agenda nenhum paciente com uma ideia prévia de 
“para onde eu vou mandar?”. Aqui não, a gente sempre pensa assim: o que a gente pode fazer? (P.6 - enfermeira, grupo 2)

O trecho de fala apresentado está em consonância com a proposta do Ministério da Saúde (Brasil, 2003), a qual preconiza que as ações de saúde mental na AB devem seguir o modelo de redes de cuidado, de base territorial. Nessa relação, deve-se considerar a noção de território, intersetorialidade, reabilitação psicossocial e interdisciplinaridade. Além disso, corresponde com a proposta de que os serviços devem encaminhar para cuidado especializado somente os casos em que a equipe da $\mathrm{AB}$ esgota os seus recursos de atenção. Tais aspectos também foram apontados em outros estudos, nos quais foi observado que o processo de corresponsabilização favoreceu a realização de parcerias e na articulação dos serviços (Hirdes, 2015; Lima \& Dimenstein, 2016; Machado \& Camatta, 2013; Tatmatsu \& Araújo, 2016).

A troca entre profissionais e serviços está no núcleo da ferramenta do AM. Assim, o AM, quando exercido em todo seu pontencial, promove maior articulação da rede da RAPS. Serviço e profissionais que possuem uma maior compreensão do trabalho em rede podem fortalecem a cultura de corresponsabilização, visto que os efeitos positivos podem ser percebidos no cotidiano dos serviços. Dessa forma, contribui-se para para que se construa e fortaleça uma rede que sirva de base para uma mudança estrutural no cuidado em saúde mental.

\section{Oferta de Atendimento em Saúde Mental}

Em relação às estratégias de cuidado frente às demandas de saúde mental, a medicação foi o recurso mais mencionado por profissionais de ambos grupos. Não obstante, nos relatos do grupo 1, esse aspecto apareceu com maior relevo, como um dos poucos recursos para tratamento. Os motivos alegados pelos entrevistados para que isso ocorra foram: alta demanda de atendimento para saúde mental, falta de recursos de atendimento, rede sobrecarregada de encaminhamentos e falta de tempo na rotina de trabalho. Esses profissionais referem que para o manejo dos casos de saúde mental seria necessário um acompanhamento de profissionais especializados, como psicólogo e psiquiatra. Diante disso, a atuação da equipe se configura no encaminhamento dos casos e na manutenção da receita do medicamento, o que assinala a vigência da estratégia medicamentosa frente a uma possível dificuldade em atender a demanda: 
Muitas vezes eu acabo repetindo uma receita que está acabando, a pessoa tem que tomar a medicação e não deu tempo de ir no seu psiquiatra renovar. É que muita coisa a unidade básica acaba absorvendo. Se a pessoa está fazendo uso de medicação, não está fazendo psicoterapia, ou ela estava na fila de espera para psiquiatria. Acaba que o profissional da atenção básica resolve muita coisa que poderia ser atendida pelo especialista. A gente atende até pelo próprio bem do paciente. (P.3 - médica, grupo 1).

Nesse fragmento de fala, percebe-se que a entrevistada não concebe a $\mathrm{AB}$ como local de ações de atenção psicossocial, mas como última alternativa. A ideia apresentada retoma a lógica de trabalho das especialidades e reflete na permanência do modelo médico centrado, o que evidencia o processo de medicalização dos sintomas que ainda persiste nos atendimentos. Sobre essa temática, são encontrados diversos estudos que sinalizam que o tratamento de usuários com sofrimento psíquico nas UBS e ESF se restringe em consultas médicas e prescrição de medicamentos (Bezerra et al., 2014; Hirdes, 2015; Moliner \& Lopes, 2013; Treichel, Onocko-Campos, \& Campos, 2018). A literatura apresenta que os profissionais compreendem a medicação como um dos únicos métodos terapêuticos para os usuários (Lima, Severo, Andrade, Soares, \& Silva, 2013; Onocko-Campos et al., 2012;), assim como o predomínio de uma visão fármaco-dependente entre os usuários (Treichel, Oncocko-Campos, \& Campos, 2018).

Conforme apontam Bezerra et al. (2014), a medicalização objetifica os sintomas aparentes dos usuários, evidenciando a doença e não a experiência dos sujeitos, com todas suas singularidades, justificada como uma dimensão mais complexa de atuação. Segundo Moliner e Lopes (2013), o enfoque privilegiado da medicalização impede que o profissional da $\mathrm{AB}$ atente para as outras questões que permeiam os sujeitos e seus sofrimentos (Moliner \& Lopes, 2013). Atribui-se a isso a dificuldade dos profissionais em lidar com situações e casos específicos de saúde mental, relacionada à falta de formação e capacitação profissional (Bezerra et al., 2014). Desse modo, apesar da ESF se constituir como uma nova proposta de reorganização de assistência à saúde, prevalece o modelo biomédico e medicalizantes da atenção à saúde mental.

Em alternativa, outros profissionais apresentam uma problematização e crítica ao tratamento medicamentoso como único recurso terapêutico, conforme o exemplo: 
Às vezes não precisa de medicação, ou daquela dosagem. Precisa de alguém para conversar. Nem que não fale, que só escute. Não é só o remédio que funciona. Não existe meio termo dentro da Atenção Básica. Ou trata com remédio, ou não trata. Mesmo que não vá ao psiquiatra, o médico da unidade pode dar uma medicação. Porque não tem tempo de ficar 45 minutos ouvindo. Às vezes ele pode querer ouvir, mas não tem tempo. Vai lá, pega tua fluoxetina, teu rivotril, teu haloperidol, o que tu tiver que tomar (...) Apenas com o remédio. E o meio termo? (P.10-ACS, grupo 1).

O fragmento de fala denota a denúncia da medicalização como principal e muitas vezes como único recurso terapêutico e a insatisfação pela falta de alternativas para tratamento na AB. O "meio termo" no qual a profissional se refere pode ser entendido como a possibilidade de haver outras ofertas de atendimento, que deveriam ser desenvolvidas tanto pela unidade, como em conjunto com a equipe matricial.

Nesse sentido, os entrevistados demonstraram em suas falas o entendimento de que a $\mathrm{AB}$ se configura enquanto locus para atendimento em saúde mental, para além do tratamento medicamentoso. Contudo, há o questionamento sobre a função que as unidades desempenham e o que pode ser feito em relação à articulação da rede de atenção psicossocial. Tais reflexões são evidenciadas em profissionais no grupo 2, conforme as falas:

Às vezes o paciente precisa de psicoterapia, precisa de algo que a gente não consegue dar conta e aí não podemos encaminhar. Não tem profissional o suficiente para suprir essa demanda. Nós acabamos atendendo aqui para não ter que levar todos. A gente acaba fazendo um trabalho que eu não digo que não seja nosso, porque ele é nosso, só que seria melhor feito por outro profissional (P.1 - enfermeira, grupo 2).

Tem usuários que não precisaria estar medicando. E para diminuir essa ansiedade, que a fala, a escuta, ou uma conversa daria... tu ficas fazendo o que podes. A gente atende muita gente da saúde mental. Consegue matriciar pouco (P.15 - médica da família, grupo 2).

$\mathrm{Na}$ fala da enfermeira, apesar de haver a compreensão de que a $\mathrm{AB}$ deve prestar cuidado em saúde mental, também existe a noção de que as especialidades poderiam disponibilizar de melhores condições de atendimento. A segunda profissional, além de evidenciar o reconhecimento da medicalização da atenção à saúde mental, demonstra a frustação frente à falta de disponibilidade na rede para atender as demandas. 
Conforme apontam Lima e Dimenstein (2016), há o reconhecimento da insuficiência do matriciamento na RAPS, mas compreende-se que a prática se encontra em processo de germinação. O apoio matricial, independentemente de qual equipe de apoio esteja envolvida, é uma importamente ferramenta para a consolidação do modelo de atenção psicossocial.

\section{Ações de Apoio Matricial em Saúde Mental}

De acordo com as recomendações do Ministério da Saúde, as principais ações de apoio matricial que podem ser desenvolvidas pelo NASF correspondem às discussões de casos, atendimento conjunto, construção conjunta de projetos terapêuticos, atividades de educação permanente, intervenções no território e na saúde de grupos populacionais, ações intersetoriais, ações de prevenção e promoção da saúde, entre outros (Portaria n. 3.088, 2011). Com isso, nas unidades pesquisadas, entre as ações de apoio matricial, a discussão de caso foi a atividade reportada com maior regularidade por todos entrevistados, independentemente de qual grupo. Esse dado também foi apontado em outra pesquisa realizada na região metropolitana de Porto Alegre (Hirdes, 2015).

Os profissionais correspondentes ao grupo 1 relataram que as ações de apoio matricial das equipes de saúde mental restringiam-se às discussões de casos com a finalidade de encaminhar usuários e raras intervenções de profissionais como psiquiatras nas unidades. Estudos demonstram que a preferência da prática de discussões de casos para realizar encaminhamentos aponta para a parcialidade da implantação do apoio matricial, pois a $\mathrm{AB}$ pode passar a funcionar apenas como porta de entrada para a atenção especializada, sendo que essa não é a sua proposta (Castro \& Campos, 2016; Onocko-Campos, et al., 2012).

De acordo com os relatos, as discussões de casos, que ocorrem nas reuniões de matriciamento, apresentaram uma variação de periodicidade de uma vez ao mês, duas vezes ao mês até uma vez por semana. Destaca-se que os serviços com apoio NASF indicaram a maior frequência de reuniões com apoiadores nas unidades.

Em relação às intervenções em grupo voltadas para a saúde mental, percebeu-se que tanto o Grupo 1 quanto o 2 realizam essas atividades. No que concerne as ações do Grupo 2, foi constatado em apenas uma unidade a participação conjunta da ESF e NASF nas suas execuções. Outra unidade recebia o auxílio do NASF para a condução dos grupos durante o matriciamento, sem sua presença efetiva durante as intervenções.

Em ambos grupos foi evidenciado um preocupação com o caráter estigmatizador que pode ser atrelado a um grupo de saúde mental. Tal aspecto pode ser observado pelas 
denominações, como "Grupo saúde do corpo e da mente", "Grupo evolução" e "Grupo de saúde comunitária" e na forma de como é realizado, com respeito aos diferentes usuários com suas singularidades, além dos mesmos possuírem um papel ativo dentro dos grupos. Destacase que em apenas três unidades não foi relatada a realização de intervenções em grupo.

Começaram a fazer brechós, os colaboradores trazem as coisas de casa e duas vezes ao mês a gente tem o brechó, e aí a gente reverte esse dinheiro para uso dos grupos. Os usuários tiveram a ideia de, por que não se autogerir? Eles poderiam estar fazendo uma feira e tal. E se envolvendo também nisso. As pessoas vêm muito (ao grupo), e está sendo um espaço de escuta e de ajuda, um espaço de confraternização (P.6 - Enfermeira, grupo 2).

Ao analisar os relatos referente ao Grupo 2, foi possível identificar mais ações de AM presentes no cotidiano das ESF. O processo de trabalho do AM do NASF foi descrito como atendimentos e intervenções conjuntas, troca entre as equipes na avaliação dos casos e na orientação das condutas. Conforme exposto, os casos permanecem sob o cuidado da equipe ESF e quando há ações específicas do NASF, a equipe ESF mantém o seguimento do cuidado de maneira complementar. Isso ocorre, por exemplo, quando os usuários são encaminhados para serviços de outros níveis de atenção.

Os encontros de matriciamento que acontecem uma vez ao mês são para discutir casos novos e também é um momento de educação continuada, tá?E, além disso, todos os profissionais do NASF tem agendas aqui dentro da equipe. Ou seja, aquele caso que foi discutido, acontece uma consulta conjunta entre o médico e o psiquiatra (P.13 - enfermeira, grupo 2.)

O exemplo de fala indica a realização de atendimentos conjuntos entre profissionais da ESF e NASF, e a utilização da discussão de casos como um espaço propício para práticas de educação permanente. Portanto, percebe-se que tais questões correspondem ao que é recomendado como atuação de apoio matricial de equipe NASF.

No entanto, considera-se que o uso do espaço para discussão de casos não deve ser excessivo, para que essa prática não assuma o papel central em detrimento de outras tarefas. Nesse sentido, estudos observaram a utilização de tempo em demasia para discutir casos ou reuniões de matriciamento com equipes $\mathrm{ESF}$, o que negligenciava a realização de outras 
aitivdades também importantes, como os atendimentos conjuntos (Gonçalves, Lancman, Sznelwar, Cordone, \& Barros, 2015).

No que tange ao desenvolvimento do Projeto Terapêutico Singular (PTS), observou-se a não realização e o desconhecimento do termo na grande maioria nos entrevistados. Tal dado converge com outros estudos, os quais indicam que a construção do PTS não faz parte do cotidiano de trabalho (Ferro, Silva, Zimmermann, Castanharo, \& Oliveira, 2014; Gonçalves et al., 2015; Hori \& Nascimento, 2014; Leite, Andrade, \& Bosi, 2013). Destaca-se que o PTS tem como objetivo a elaboração de estratégias de intervenção, utilizando recursos da equipe interdisciplinar, da família, da comunidade e do próprio sujeito (Hori \& Nascimento, 2014). Em apenas uma unidade foi relatada a execução do PTS de forma congruente, ou seja, com a participação do usuário como agente ativo no processo.

O PTS, enquanto uma uma tecnologia leve de cuidado (Mehry, 2002), se mostra fundamental para a produção de cuidado integral em saúde mental, uma vez que pode facilitar a aproximação entre equipe/usuário, a permanência do usuários no serviço e no tratamento. Assim, o uso dessa ferramenta permite pensar o cuidado de maneira articulada com demais setores da vida dos sujeitos, sejam eles, sociais, econômicos, psicológicos, biológicos, etc (Jorge, Diniz, Lima, \& Penha, 2015). Dessa forma, a partir desse diálogo é possível produzir um cuidado alinhado à singularidade dos usuários, o que promove uma compreensão sobre o que é experienciado por cada sujeito, potencializando o manejo da demanada e do estabelecimento de ações de saúde mental mais próximas do cotidiano de cada um.

\section{Avaliação dos Profissionais do Apoio Matricial em Saúde Mental}

Dos dez entrevistados correspondentes ao Grupo 2, nove avaliam positivamente em algum aspecto o apoio matricial da equipe NASF. Os entrevistados percebem o apoio matricial como uma estratégia importante para lidar com situações de saúde mental na $\mathrm{AB}$. Ademais, os profissionais reconhecem a relevância do trabalho da equipe NASF na qualificação do atendimento em saúde mental oferecido nas unidades. Os benefícios associados são referentes ao processo de encaminhamento para a atenção especializada, na corresponsabilização dos casos e também na possibilidade de contar com a retaguarda especializada na condução de ações no território:

Desde que mudou essa forma de encaminhar, de fazer o matriciamento... é trabalhoso. Porém, eu acredito que seja melhor. Chegar e conversar com a equipe, 
passar a situação do paciente, às vezes atender junto... nós conseguimos ser mais resolutivos. Melhorou, na questão de qualificar o encaminhamento.. Porém, eu acredito que teria a necessidade de ampliar, de poder ter uma oferta maior e atender melhor (P.1 - enfermeira, Grupo 2).

Eu não sei como eu trabalhava antes sem o apoio do NASF! Ter esse suporte, um colega especialista com quem posso trocar. Claro que não são todos os casos, e, com o tempo, a ideia é andar com as próprias pernas, não é o NASF vir atender para nós. Eu acho que hoje é o que dá característica para os serviços da atenção básica realmente abraçarem a causa da saúde mental (P.6 - enfermeira, Grupo 2).

A fala da primeira profissional expressa a capacidade do trabalho do NASF de atuar no rompimento da lógica de encaminhamento. Essa ruptura se reflete na oposição ao mecanismo fragmentado de cuidado. Se uma das dificuldades da RAPS se encontra na efetivação das linhas de cuidado, que apresentam fluxos fragmentados, compreende-se isso como o grande efeito do apoio matricial do NASF: a diminuição dos encaminhamentos desnecessários. Por outro lado, destaca-se ainda a importância de ampliação da rede especializada para os casos que necessitem deste acompanhamento, pois a ideia não é a $\mathrm{AB}$ atender todas as demandas, o tempo todo. No segundo fragmento de fala, as ações e o cuidado no território são atribuídos ao trabalho de sensibilização dos apoiadores do NASF para as questões de saúde mental na $\mathrm{AB}$, o que representa também um grande potencial que os profissionais dos núcleos possuem.

Sobre esses aspectos, a literatura refere que o apoio matricial do NASF propicia a construção coletiva de saberes em saúde mental, fomentando a implantação de redes do cotidiano, possibilitando, dessa forma, uma maior aceitação e compreensão da saúde mental na $\mathrm{AB}$ aos serviços e aos profissionais (Morais \& Tanaka, 2012; Tatmatsu \& Araújo, 2016; Amaral, Torrenté, Torrenté, \& Moreira, 2018). Outras pesquisas avaliaram que a implantação do apoio matricial impactou na resolutividade das equipes, na ampliação do acesso da população aos serviços, através da diminuição do número de encaminhamentos, possibilitando acordos de classificação de risco e organização de fluxos. (Lima \& Dimenstein, 2016; Machado \& Camatta, 2013; Santos \& Lacaz, 2012).

Com isso, é passível de reflexão de que se um dos objetivos do NASF é atuar diretamente na lógica de cuidado compartilhado, dando suporte para as equipes da $\mathrm{AB}$ na atenção em saúde mental, pode-se compreender que nos serviços pesquisados essa meta vem sendo alcançada em certa medida. Em contrapartida, os profissionais que atuam em unidades 
sem apoio do NASF, ou seja, correspondentes ao grupo 1, não compartilham dessa mesma perspectiva. Nota-se que nesses serviços estudados, a concepção de que a AB faz parte da rede de atenção psicossocial não está consolidada:

Eu acho que poderia ter um centro de referência para isso. O ideal seria que o usuário fosse direto no profissional que está necessitando. Aqui na Atenção Básica, ele vai ser encaminhado para todas as especialidades. Para a psiquiatria, se tivesse, cortaria caminho para o próprio usuário (...) Faltam profissionais, falta um local só para isso (P.8 - enfermeira, Grupo 1).

Pelo o pouco que eu tenho notado, o apoio matricial é uma relação que funciona em termos. A gente leva o caso e precisa de acompanhamento com psiquiatra, mas só tem consulta daqui há não sei quanto tempo. E até então, como fica esse paciente? Nesse ponto, eu vou ser bem sincera, eu me sinto abandonada. Como profissional, e até mesmo como paciente, eu gostaria de ter ajuda do NASF. Às vezes eu me sinto perdida (P.10-ACS, Grupo 1).

Sendo assim, o apoio matricial nas unidades com NASF parece ter produzido uma maior corresponsabilização pelos casos, evidenciado pelo entendimento das equipes de que contam com uma retaguarda especializada adequada. Nas unidades com apoio de equipe de referência, as ações de matriciamento, muitas vezes, limitaram-se à discussão de casos ou a definição de encaminhamentos, favorecendo a percepção de estarem sozinhos para lidar com a complexidade da saúde mental.

Por outro lado, compreende-se também que as dificuldades e barreiras do apoio matricial das equipes de referência de saúde mental vão além dos processos de trabalho e não dependem apenas do tipo de apoio matricial recebido, mas envolvem também importantes questões estruturais, como falta de recursos humanos para atender a intensa demanda da $\mathrm{AB}$. A natureza dos tipos de equipe também merece destaque. As equipes NASF, que pertencem a $\mathrm{AB}$, por serem recentes e também itinerantes, podem ter contribuído para percepções e ações diferentes nos contextos pesquisados. As equipes de referência, que atuam a nível secundário, precisam lidar com um maior número de serviços da rede, o que pode dificultar a absorção dos princípios e processos de trabalho da AB. Desse modo, destaca-se a necessidade de desenvolver não só ações de apoio matricial nos territórios, mas de ampliar todos os tipos de equipe, que atendem um elevado número de serviços, o que compromete a articulação da rede de atenção psicossocial. 


\section{Considerações Finais}

Os problemas de saúde mental da população e a suas complexidades requerem a articulação das redes e formas assistenciais as quais os usuários recorrem para suas demandas e necessidades. No contexto da $\mathrm{AB}$, o território se configura como o lugar onde as situações e as relações mais complexas se fazem presentes. Nessa perspectiva, destaca-se o apoio matricial como importante estratégia para o desenvolvimento e efetivação de práticas de saúde mental integrais.

A partir da realização desse estudo, foi possível encontrar aproximações nas práticas de apoio matricial, bem como perceber alguns distanciamentos sobre os processos de trabalho nos dois grupos de profissionais. No que concerne às diferenças das modalidades de apoio matricial, analisa-se que as ações do NASF são descritas pelos entrevistados de forma mais detalhada e diversificada, enquanto que no grupo 1, o apoio matricial aparece descrito de forma superficial, com pouca integração entre as equipes e poucas intervenções no território. Ademais, foi possível perceber que uma das principais diferenças encontra-se na maior presença das Equipes NASF nas unidades básicas, atuando no território, o que contribui para a construção de ações conjuntas com a equipe ESF. Nesse sentido, o apoio matricial tem proporcionado efeitos na dimunição dos encaminhamentos indevidos, maior participação e integração das equipes da $\mathrm{AB}$ nas ações de saúde mental, e consequentente, maior efetividade e resolutibilidade da atenção à saúde mental dos usuários.

Compreende-se que o NASF é um recurso imprescindível que tem mostrado efeitos e resultados significativos na melhoria da atenção à saúde mental. Destaca-se que a disponibilidade das equipes de $\mathrm{AB}$ contarem com a retaguarda do NASF ainda não corresponde a uma realidade de todos serviços de Porto Alegre. Salienta-se a necessidade de ampliar as equipes de NASF, com ações voltadas para a educação permanente e capacitações em saúde mental.

Apesar de haverem avanços no que consiste o apoio matricial na $\mathrm{AB}$, algumas ações ainda são pouco ou pacialmente desenvolvidas, o que pode comprometer ou ser um dificultador do processo de cuidado em saúde mental nos serviços. Assim, é importante qualificar o apoio matricial de todas equipes, tanto de NASF como de equipes de saúde mental. A intenção não é desqualificar o apoio matricial realizado por equipes de referência, mas sim destacar a importância do estabelecimento de medidas e ações que busquem consolidar todas as modalidades de equipe de apoio matricial enquanto estratégia de qualificação da atenção à saúde mental na $\mathrm{AB}$. Consolidar avanços, promover e ampliar novas 
ações de saúde mental exigem uma integração usuários e profissionais na defesa das conquistas da atenção psicossocial.

Como limitação do estudo, detaca-se que a pesquisa apresenta um recorte da situação da ações de saúde mental no nível da $\mathrm{AB}$ de uma cidade de grande porte. Apesar disso, considera-se como potencialidade do estudo a profícua reflexão acerca da $\mathrm{AB}$ no que diz respeito aos cuidados à saúde mental, principalmente em relação à importância do apoio matricial enquanto estratégia para a maior resolutividade e efetividade do cuidado. Os resultados apresentados e discutidos apontam para questões relevantes que podem servir como norteadoras de políticas públicas de saúde mental. Com o intuito de ampliar os temas abordados, sugere-se a realização de novos estudos que levem em consideração a perspectiva de outros atores envolvidos, como os usuários dos serviços.

\section{Referências}

Alvarez, A. P. E., Vieira, Á. C. D., \& Almeida, F. A. (2019). Núcleo de Apoio à Saúde da Família e os desafios para a saúde mental na atenção básica. Physis: Revista de Saúde Coletiva, 29(4), 1-17. doi:10.1590/s0103-73312019290405

Amaral C. E. M., Torrenté, M. O. N., Torrenté, M., \& Moreira, C. P. (2018). Apoio matricial em saúde mental na atenção básica: Efeitos na compreensão e manejo por parte de agentes comunitários de saúde. Interface (Botucatu), 22(66), 801-12. Recuperado de https://pesquisa.bvsalud.org/portal/resource/pt/biblio-954303

Bezerra, I. C., Jorge, M. S. B., Gondim, A. P. S., Lima, L. L., \& Vasconcelos, M. G. F. (2014). "Fui lá no posto e o doutor me mandou foi pra cá": Processo de medicamentalização e (des)caminhos para o cuidado em saúde mental na Atenção Primária. Interface - Comunicação, Saúde, Educação, 18(48), 61-74. doi:10.1590/1807-57622013.0650

Bonfim, G. I., Bastos, E. N. E., Góis, C. W. L., \& Tófoli, L. F. (2013). Apoio matricial em saúde mental na atenção primária à saúde: Uma análise da produção científica e documental. Interface - Comunidade, Saúde, Educação, 17(45), 287-300. doi:10.1590/S1414-32832013005000012

Brasil (2001). Lei n. 10.216, de 6 de abril de 2001. Dispõe sobre a proteção e os direitos da pessoas portadoras de transtornos mentais e redireciona o modelo assistencial em saúde mental. Brasília, DF: Ministério da Saúde. Recuperado em http://www.planalto.gov.br/ccivil_03/leis/leis_2001/110216.htm 
Brasil. (2003). Saúde mental e atenção básica - o vínculo e o diálogo necessários - inclusão das ações de saúde mental na atenção básica. Brasília, DF: Ministério da Saúde, Secretaria de Atenção à Saúde.

Brasil (2008). Portaria n. 154, de 24 de janeiro de 2008. Cria os Núcleos de Apoio à Saúde da Família - NASF. Brasília, DF: Ministério da Saúde. Recuperado de http://bvsms.saude.gov.br/bvs/saudelegis/gm/2008/prt0154_24_01_2008.html

Brasil (2011). Portaria n. 3.088, de 23 de dezembro de 2011. Institui a Rede de Atenção Psicossocial para pessoas com sofrimento ou transtorno mental e com necessidades decorrentes do uso de crack, álcool e outras drogas, no âmbito do Sistema Único de Saúde (SUS). Brasília, DF: Ministério da Saúde. Recuperado em http://bvsms.saude.gov.br/bvs/saudelegis/gm/2011/prt3088_23_12_2011_rep.html

Brasil. (2013). Cadernos de Atenção Básica: Saúde Mental, 34. Brasília, DF: Ministério da Saúde. Recuperado de http://bvsms.saude.gov.br/bvs/publicacoes/cadernos_atencao_basica_34_saude_menta 1

Brasil (2017). Portaria n. 2.436, de 21 de setembro de 2017. Aprova a Política Nacional de Atenção Básica, estabelecendo a revisão de diretrizes para a organização da Atenção Básica, no âmbito do Sistema Único de Saúde (SUS). Brasília, DF: Ministério da Saúde. http://bvsms.saude.gov.br/bvs/saudelegis/gm/2017/prt2436_22_09_2017.html

Braun, V., \& Clarke, V. (2006). Using thematic analysis in psychology. Qualitative Research in Psychology, 3(2), 77-101. Recuperado de https://www.tandfonline.com/doi/abs/10.1191/1478088706qp063oa

Castro, C. P., \& Campos, G. W. S. (2016). Apoio Matricial como articulador das relações interprofissionais entre serviços especializados e atenção primária à saúde. Physis: Revista de Saúde Coletiva, 26(2), 455-481. doi:10.1590/S0103-73312016000200007

Castro, C. P., Oliveira, M. M., \& Campos, G. W. S. (2016). Apoio Matricial no SUS Campinas: Análise da consolidação de uma prática interprofissional na rede de saúde. Ciência \& Saúde Coletiva, 21(5), 1625-1636. doi:10.1590/141381232015215.19302015

CNES. (2017). Cadastro Nacional dos Estabelecimentos de Saúde do Brasil. Brasília, DF: Ministério da Saúde. 
Costa, F. R. M., Lima, V. V., Silva, R.F., \& Fioroni, L. N. (2015). Desafios do apoio matricial como prática educacional: A saúde mental na atenção básica. Interface Comunicação, Saúde, Educação, 19(54), 491-502. doi:10.1590/1807-57622013.0816

Eslabão, A. D., Santos, E. O., Santos, V. C. F., Rigatti, R., Mello, R. M., \& Schneider, J. F. (2019). Saúde mental na estratégia saúde da família: Caminhos para uma assistência integral em saúde. J. Nurs. Health, 9(1), 1-23. doi:10.15210/JONAH.V9I1.11106

Ferro, L. F., Silva, E. C., Zimmermann, A. B., Castanharo, R. C. T., \& Oliveira, F. R. L. (2014). Interdisciplinaridade e intersetorialidade na Estratégia Saúde da Família e no Núcleo de Apoio à Saúde da Família: Potencialidades e desafios. O Mundo da Saúde, 38(21), 129-138. Recuperado de https://pesquisa.bvsalud.org/portal/resource/pt/mis36869? lang=pt

Gerbaldo, T. B., Arruda, A. T., Horta, B. L., \& Garnelo, L. (2018). Avaliação da Organização do Cuidado em Saúde Mental na Atenção Básica à Saúde do Brasil. Trabalho, Educação e Saúde, 16(3), 1079-1094. doi:10.1590/1981-7746-sol00150

Gonçalves, R. M. A., Lancman, S., Sznelwar, L. I., Cordone, N. G., \& Barros, J. O. (2015). Estudo do trabalho em Núcleos de Apoio à Saúde da Família (NASF), São Paulo, Brasil. Revista brasileira de Saúde ocupacional, 40(131), 59-74. doi:10.1590/03037657000078013

Hirdes, A. (2015). A perspectiva dos profissionais da Atenção Primária à Saúde sobre o apoio matricial em saúde mental. Ciência \& Saúde Coletiva, 20(2), 371-382. doi:10.1590/1413-81232015202.11122014

Hori, A. A., \& Nascimento, A. F. (2014). O Projeto Terapêutico Singular e as práticas de saúde mental nos Núcleos de Apoio à Saúde da Família (NASF) em Guarulhos (SP), Brasil. Ciência \& Saúde Coletiva, 19(8), 3561-3571. doi:10.1590/141381232014198.11412013

Jorge, M. S. B., Diniz, A. M., Lima, L. L., \& Penha, J. C. (2015). Apoio matricial, projeto terapêutico singular e produção do cuidado em saúde mental. Texto \& Contexto Enfermagem, 24(1), 112-120. doi:10.1590/0104-07072015002430013

Klein, A. P., \& d'Oliveira, A. F. P. L. (2017). O "cabo de força" da assistência: Concepção e prática de psicólogos sobre o Apoio Matricial no Núcleo de Apoio à Saúde da Família. Cadernos de Saúde Pública, 33(1), 1-10. doi:10.1590/0102-311x00158815

Leite, D. C., Andrade, A. B., \& Bosi, M. L. M. (2013). A inserção da Psicologia nos Núcleos de Apoio à Saúde da Família. Physis Revista de Saúde Coletiva, 23(4), 1167-1187. doi:10.1590/S0103-73312013000400008 
Lima, M., \& Dimenstein, M. (2016). O apoio matricial em saúde mental: Uma ferramenta apoiadora da atenção à crise. Interface (Botucatu), 20(58), 625-635. doi:10.1590/180757622015.0389

Lima, A. I. O., Severo, A. K., Andrade, N. L, Soares, G. P., \& Silva, L. M. (2013). O desafio da construção do cuidado integral em saúde mental no âmbito da atenção primária. Temas em Psicologia, 21(1), 71-82. doi:10.9788/TP2013.1-05

Machado, D. K. S., \& Camatta, M. W. (2013). Apoio matricial como ferramenta de articulação entre a Saúde Mental e a Atenção Primária à Saúde. Cadernos Saúde Coletiva, 21(2), 224-232. Recuperado de http://www.scielo.br/pdf/cadsc/v21n2/18.pdf

Merhy, E. E. (2002). Saúde: A cartografia do trabalho vivo. São Paulo: Hucitec.

Moliner, J., \& Lopes, S. M. B. (2013). Saúde mental na atenção básica: Possibilidades para uma prática voltada para a ampliação e integralidade da saúde mental. Saúde $e$ Sociedade, 22(4), 1072-1083. doi:10.1590/S0104-12902013000400010

Morais, A. P. P., \& Tanaka, O. Y. (2012). Apoio matricial em saúde mental: Alcances e limites na atenção básica. Saúde e Sociedade, 21(1), 161-170. doi:10.1590/S010412902012000100016

Onocko-Campos, R. T., Campos, G. W. S., Ferrer, A. L., Corrêa, C. R. S., Madureira, P. R., Gama, C. A. P., Dantas, D. V., \& Nascimento, R. (2012). Avaliação de estratégias inovadoras na organização da Atenção Primária à Saúde. Revista de Saúde Pública, 46(1), 43-50. doi:10.1590/S0034-89102011005000083

Porto Alegre (2014). Plano Municipal de Saúde 2014-2017. Porto Alegre: Secretaria $\begin{array}{llll}\text { Municipal de } & \text { Saúde. }\end{array}$ http://www2.portoalegre.rs.gov.br/sms/default.php?p_secao=927

Santos, A. P. L., Lacaz, F. A. C. (2012). Apoio matricial em saúde do trabalhador: Tecendo redes na atenção básica do SUS, o caso de Amparo/ SP. Ciência \& Saúde Coletiva, 17(5), 1143-1150. doi:10.1590/S1413-81232012000500008

Santos, R. A. B. G., Uchôa-Figueiredo, L. R., \& Lima, L. C. (2017). Apoio matricial e ações na atenção primária: Experiência de profissionais de ESF e Nasf. Saúde em Debate, 41(114), 694-706. doi:10.1590/0103-1104201711402

Tatmatsu, D. B., \& Araújo, A. C. (2016). Atenção primária e saúde mental: Contribuições e potencialidades do apoio matricial. Mudanças, 24(2), 71-79. doi:10.15603/21761019/mud.v24n2p71-79 
Treichel, C. A. S., Onocko-Campos, R. T., \& Campos, G. W. S. (2019). Impasses e desafios para consolidação e efetividade do apoio matricial em saúde mental no Brasil. Interface - Comunicação, Saúde, Educação, 23, 1-18. doi:10.1590/interface.180617

\section{Endereço para correspondência}

\section{Larissa Moraes Moro}

Avenida Ipiranga, 6681, prédio 11 sala 931, Partenon, Porto Alegre - RS, Brasil. CEP 90619-900

Endereço eletrônico: larisssamoraesmoro@gmail.com

\section{Guilherme Severo Ferreira}

Avenida Ipiranga, 6681, prédio 11 sala 931, Partenon, Porto Alegre - RS, Brasil. CEP 90619-900

Endereço eletrônico: guilherme.ferreiraa@gmail.com

\section{Kátia Bones Rocha}

Avenida Ipiranga, 6681, prédio 11 sala 931, Partenon, Porto Alegre - RS, Brasil. CEP 90619-900

Endereço eletrônico: katiabonesrocha@gmail.com

Recebido em: 10/08/2019

Reformulado em: 09/03/2020

Aceito em: 16/03/2020

\section{Notas}

* Psicóloga, Mestre em Psicologia Social e doutoranda no Programa de Pós-Graduação em Psicologia da PUCRS.

** Psicólogo de um Centro de Referência de Assistência Social. Mestre pelo Programa de Pós-Graduação em Psicologia da PUCRS.

*** Professora do Programa de Pós-Graduação em Psicologia da PUCRS. Doutora em Psicologia pela Universidad Autónoma de Barcelona.

Financiamento: CNPq.

Este artigo de revista Estudos e Pesquisas em Psicologia é licenciado sob uma Licença Creative Commons Atribuição-Não Comercial 3.0 Não Adaptada. 